\title{
Expression of Tspan-1 gene in patients with advanced gastric cancer
}

\author{
YAOWU CAI $^{1}$, MAOSONG ZHENG ${ }^{1}$, ZHIQIANG ZHAO ${ }^{1}$, HANXING HUANG $^{2}$, WENDA FU $^{2}$ and XUEFENG XU ${ }^{1}$ \\ Departments of ${ }^{1}$ Gastrointestinal Surgery and ${ }^{2}$ Pathology, The First Hospital of Putian, \\ Teaching Hospital of Fujian Medical University, Putian, Fujian 351100, P.R. China
}

Received February 27, 2017; Accepted June 19, 2017

DOI: $10.3892 / 01.2017 .6529$

\begin{abstract}
The present study investigated the correlations of the Tspan-1 gene expression with the clinical characteristics and survival prognoses of patients with advanced gastric cancer. A total of 150 patients with advanced gastric cancer were enrolled in the present study, of whom 84 were at stage II and 66 were at stage III according to the tumor node metastasis (TNM) staging; the immunohistochemical staining method and the semi-quantitative PCR method were used to detect the positive expression rates and mRNA relative expression levels of Tspan-1, vascular endothelial growth factor (VEGF), E-cadherin and N-cadherin. The positive expression rates of Tspan-1, VEGF, E-cadherin and $\mathrm{N}$-cadherin were $58.0 \%$ (87 patients), $50.0 \%$ (75 patients), $28.0 \%$ (42 patients) and $53.3 \%$ (80 patients), respectively. The positive expressions and mRNA levels of Tspan-1, VEGF, E-cadherin and $\mathrm{N}$-cadherin were not correlated with sex or age $(\mathrm{P}>0.05)$, but associated with the cancer state (stage II or stage III) and maximum tumor diameter $(\mathrm{P}<0.05)$. With the increase of stage and tumor diameter, the positive rates and mRNA levels of Tspan-1, VEGF and N-cadherin were increased, while those of E-cadherin were decreased. Among patients with stage II/III advanced gastric cancer, those with positive expression of Tspan-1, VEGF and N-cadherin had lower median survival time and survival rates than patients with negative expressions, while patients with positive expression of E-cadherin had higher median survival time and survival rate than those with negative expression $(\mathrm{P}<0.05)$. The high expression of Tspan-1 gene is associated with the TNM staging of advanced gastric cancer and the tumor diameter, influences the survival prognosis, and may involve the processes of angiogenesis and epithelial-mesenchymal transition.
\end{abstract}

Correspondence to: Dr Xuefeng Xu, Department of Gastrointestinal Surgery, The First Hospital of Putian, Teaching Hospital of Fujian Medical University, 449 Nanmen West Road, Putian, Fujian 351100, P.R. China

E-mail: xuefengxu2016@163.com

Key words: Tspan-1 gene, advanced gastric cancer, angiogenesis, epithelial-mesenchymal transition

\section{Introduction}

As the most common gastrointestinal malignancy, gastric cancer ranks fourth in incidence and second only to lung cancer in mortality among all malignant tumors (1). There is an absence of early specific clinical manifestations of patients with advanced gastric cancer; therefore, approximately 30\%-70\% of patients are newly diagnosed with advanced gastric cancer and the 3-year recurrence and metastasis rates of early gastric cancer cells after operation are approximately 40\%-80\% (2). The present study demonstrated that the occurrence and development of gastric cancer cells is a multi-gene involved multi-stage process, and the prognosis is closely related to tumor-node-metastasis (TNM) staging, but is different in patients with same-stage gastric cancer (3). Looking for molecular markers with high sensitivity and specificity is of great significance to improve the early diagnosis, treatment and prognosis. Tspan-1 is a member of transmembrane-4 superfamily (TM4SF) of proteins, is mainly located in the cell membrane, has a common phenomenon of glycosylation and plays important roles in intercellular adhesion, invasion and metastasis (4). Overexpression of Tspan-1 is found in ovarian cancer (5), colorectal cancer (6), hepatocellular carcinoma (7), cervical cancer (8), breast cancer (9), pancreatic cancer (10) and glioma (11), and is closely correlated with clinical features, therapeutic efficacy and survival prognoses of tumors. Tspan- 1 gene in vitro can regulate and control the proliferation, differentiation, invasion, apoptosis, angiogenesis and other behaviors of tumor cells (12). A few recent studies have also pointed out that $(13,14)$, the abnormal expression of Tspan-1 gene may be closely associated with the occurrence and development of gastric cancer cells. The present study analyzed the roles of Tspan-1 expression in the processes of angiogenesis and epithelial-mesenchymal transition (EMT), study whether it is associated with clinical features and prognoses of patients with advanced gastric cancer, to provide new targets for clinical evaluation of diagnosis, treatment and prognosis.

\section{Patients and methods}

A total of 150 patients diagnosed with advanced gastric cancer and admitted to the First Hospital of Putian from January 2013 to June 2016 were continuously selected and pathological diagnosis was confirmed. Among them were males $(n=78)$ and females $(n=72)$, with an average age of $62.5 \pm 15.6$ years and a 
mean maximum tumor diameter of $3.3 \pm 1.4 \mathrm{~cm}$. Patients were at stage II $(n=84)$ and stage III $(n=66)$, according to the TNM staging. Informed consent was obtained from the individuals who participated in the research. The present study was approved by the Ethics Committee at the First Hospital of Putian.

Study methods and observation indicators. A therapeutic regimen recommended by standard medical guidelines was used, i.e., a combination of surgical therapy, radiotherapy and chemotherapy and targeted therapy. Immunohistochemical staining method and semi-quantitative PCR method were used to detect the positive expression rates and mRNA relative expression levels of Tspan-1, vascular endothelial growth factor (VEGF), E-cadherin and $\mathrm{N}$-cadherin. The follow-up time was 3.0 to 45.0 months and the median time was 25.0 months. The median survival time and survival rate were recorded. Related data were collected, entered and analyzed by a third party.

Immunohistochemical staining method. The following were purchased: Low temperature deep refrigerators (Haier Group, Qingdao, China), runner histotomes (LEICA RM2245; Leica Microsystems, Wetzlar, Germany), microscopes (Olympus BX51; Olympus Corporation, Tokio, Japan), pathology tissue bleaching and baking processors (TKY-TK; Hubei, China), electric-heated thermostatic hot air ovens (303-3; Shanghai, China), mouse anti-human Tspan-1, VEGF, E-cadherin and N-cadherin monoclonal antibodies (Beyotime Biotech, Jiangsu, Japan), rabbit anti-mouse IgG antibodies (Zhongshan Golden Bridge Biological Co., Ltd., Beijing, China), and PV-9000 second-generation general-purpose two-step immunohistochemical detection kits (Sigma, St. Louis, MO, USA).

Tissue sections were prepared through a routine fabrication method, with a thickness of $5 \mu \mathrm{m}$, de-waxed in xylene, rehydrated in gradient alcohol, then antigen retrieval, adding $3 \% \mathrm{H}_{2} \mathrm{O}_{2}$ solution and incubation at $27^{\circ} \mathrm{C}$ for $20 \mathrm{~min}$, and then normal goat serum working solution added dropwise and incubated at $27^{\circ} \mathrm{C}$ for $30 \mathrm{~min}$. The sections were incubated with primary antibodies $(1: 2,000)$ overnight at $4^{\circ} \mathrm{C}$ in a humidified chamber. A negative control was designed by using normal mouse IgG instead of primary antibody. Then, the sections were added with $\mathrm{IgG}$ secondary antibodies dropwise (1:500) and incubated for $20 \mathrm{~min}$ at $27^{\circ} \mathrm{C}$ in the humidified chamber; horseradish-peroxidase-labeled pronase avidin (Biyuntian Science \& Technology Co., Ltd., Jiangsu, China) were added dropwise and the sections were incubated for $20 \mathrm{~min}$ at $27^{\circ} \mathrm{C}$ in the humidified chamber and oscillated then washed with PBS for 5 min $x 3$ times. The sections were developed with DAB (diaminobenzidine), counterstained with hematoxylin, differentiated with hydrochloric alcohol, blued with ammonia, rehydrated in gradient alcohol, hyalinized in xylene, sealed with a neutral gum, dried at room temperature, and observed with an optical microscope. Result determination: the semi-quantitative method based on both the staining intensity and the proportion of stained cells was used; it was positive if the cytoplasm or nucleus was stained dark brown from yellow. The staining intensity was scored as: 0 , negative; 1 , weak; 2 , moderate; 3 , strong. The proportion of stained cells was scored as: $0, \leq 5 \% ; 1,6-25 \%$; $2,26-50 \% ; 3,51-75 \% ; 4,>75 \%$. If the product of the above two scores was $0-3$, it was considered negative, and if $4-12$, it was considered positive.

PCR method. Total RNA was extracted from cells according to a conventional method using TRIzol reagents. Concentration and purity were measured by using ultraviolet spectrophotometers. cDNA was synthesized by using reverse transcription kits. The primer sequences were synthesized by Sangon Biotech Co., Ltd. (Shanghai, China) based on gene bank sequences were: Tspan-1: forward, 5'-GGTTTCATCCAGGA TCGAGCAGG-3' and reverse, 5'-ACAAAGATGGTCACGGT CTGCC-3', 445 bp; VEGF: forward, 5'-ACTACTTCTCCCGC CGC TAC-3', and reverse, 5'-GAAATCAAACAGAGGCCGC ATG-3', 332 bp; E-cadherin: forward, 5'-ATCAAAGGTATC ACGGCAAACG-3' and reverse, 5'-CGGAGAGCTCGTCC ACG TAT-3', 479 bp; N-cadherin: forward, 5'-GTGCCATTA GCCAAGGGAATTCAGC-3', and reverse, 5'-GCGTTCCTGT TCCACTCATAGGAG-3', 337 bp; GAPDH forward, 5'-CGC GAGAAGATGACCCAGAT-3', and reverse, 5'-GCACTGTG TTGGCGTACAGG-3', $225 \mathrm{bp}$. The reaction system was $2 \mu \mathrm{l}$ cDNA $+3 \mu l$ upper primers and $3 \mu 1$ lower primers $+0.5 \mu 1$ Taq polymerase $+1 \mu \mathrm{ldNTPs}+3 \mu \mathrm{l} \mathrm{MgCl}_{2}+5 \mu \mathrm{l} 10 \mathrm{X}$

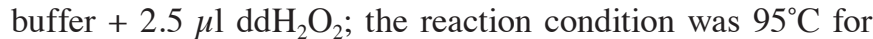
$5 \mathrm{~min}$, then $95^{\circ} \mathrm{C}$ for $30 \mathrm{sec}, 58^{\circ} \mathrm{C}$ for $30 \mathrm{sec}$ and $72^{\circ} \mathrm{C}$ for $60 \mathrm{sec}$, with a total of 30 cycles, and lastly, $72^{\circ} \mathrm{C}$ for $10 \mathrm{~min}$. PCR products were identified by $2 \%$ agarose gel electrophoresis, ultraviolet spectrometry images were formed by a gel documentation and analysis system, and gray values of digital photos were analyzed. The results were expressed by using the $2^{-\Delta \Delta \mathrm{Cq}}$ method.

Statistical analysis. SPSS 20.0 software (IBM, Armonk, NY, USA) was used for statistical analysis. Measurement data were expressed as mean \pm standard deviation and comparisons between groups were done by using independent sample $\mathrm{t}$-test; enumeration data were indicated as case or percentage (\%) and comparisons between groups were carried out by using $\chi^{2}$ test; Kaplan-Meier model and log-rank $\chi^{2}$ test were used for the median survival time; Pearson or $\chi^{2}$ test was used for the correlation analysis. $\mathrm{P}<0.05$ was considered to indicate a statistically significant difference.

\section{Results}

Analysis of immunohistochemical results. The positive expression rates of Tspan-1, VEGF, E-cadherin and N-cadherin were $58.0 \%$ (87 patients), $50.0 \%$ (75 patients), $28.0 \%$ (42 patients) and $53.3 \%$ (80 patients), respectively. The positive expressions of Tspan-1, VEGF, E-cadherin and N-cadherin was not correlated with sex or age $(\mathrm{P}>0.05)$, but associated with the cancer state (stage II or stage III) and maximum tumor diameter $(\mathrm{P}<0.05)$, that is, with the increase of stage and diameter, the positive rates of Tspan-1, VEGF and N-cadherin were increased, while that of E-cadherin was decreased (Fig. 1 and Table I).

Analysis of PCR results. The mRNA expression levels of Tspan-1, VEGF, E-cadherin and N-cadherin were not correlated with sex and age $(\mathrm{P}>0.05)$, but associated with the cancer state (stage II or stage III) and maximum tumor diameter $(\mathrm{P}<0.05)$. With the increase of cancer stage and 
Table I. Analysis of immunohistochemical results.

\begin{tabular}{|c|c|c|c|c|c|c|c|c|}
\hline \multirow[b]{2}{*}{ Item } & \multicolumn{2}{|c|}{ Tspan-1 } & \multicolumn{2}{|c|}{ VEGF } & \multicolumn{2}{|c|}{ E-cadherin } & \multicolumn{2}{|c|}{ N-cadherin } \\
\hline & $\begin{array}{l}\text { Positive } \\
(n=87)\end{array}$ & $\begin{array}{l}\text { Negative } \\
(n=63)\end{array}$ & $\begin{array}{l}\text { Positive } \\
(n=75)\end{array}$ & $\begin{array}{l}\text { Negative } \\
(\mathrm{n}=75)\end{array}$ & $\begin{array}{l}\text { Positive } \\
(n=42)\end{array}$ & $\begin{array}{l}\text { Negative } \\
(n=108)\end{array}$ & $\begin{array}{l}\text { Positive } \\
(n=80)\end{array}$ & $\begin{array}{l}\text { Negative } \\
(n=70)\end{array}$ \\
\hline Male/female & $42 / 45$ & $36 / 27$ & $40 / 35$ & $38 / 37$ & $25 / 17$ & $53 / 55$ & $46 / 34$ & $32 / 38$ \\
\hline Age, years & $59.8 \pm 13.9$ & $64.4 \pm 16.7$ & $62.3 \pm 15.8$ & $63.8 \pm 14.2$ & $63.5 \pm 12.9$ & $61.2 \pm 15.6$ & $64.9 \pm 17.2$ & $60.2 \pm 13.5$ \\
\hline Stage II/III & $40 / 47$ & $44 / 19$ & $35 / 40$ & $49 / 26$ & $30 / 12$ & $54 / 54$ & $37 / 43$ & $47 / 23$ \\
\hline $\begin{array}{l}\text { Maximum tumor } \\
\text { diameter, } \mathrm{cm}\end{array}$ & $3.9 \pm 1.6$ & $3.1 \pm 1.2$ & $3.8 \pm 1.7$ & $3.2 \pm 1.3$ & $3.1 \pm 1.3$ & $3.7 \pm 1.6$ & $4.1 \pm 2.1$ & $2.8 \pm 1.3$ \\
\hline
\end{tabular}

VEGF, vascular endothelial growth factor.
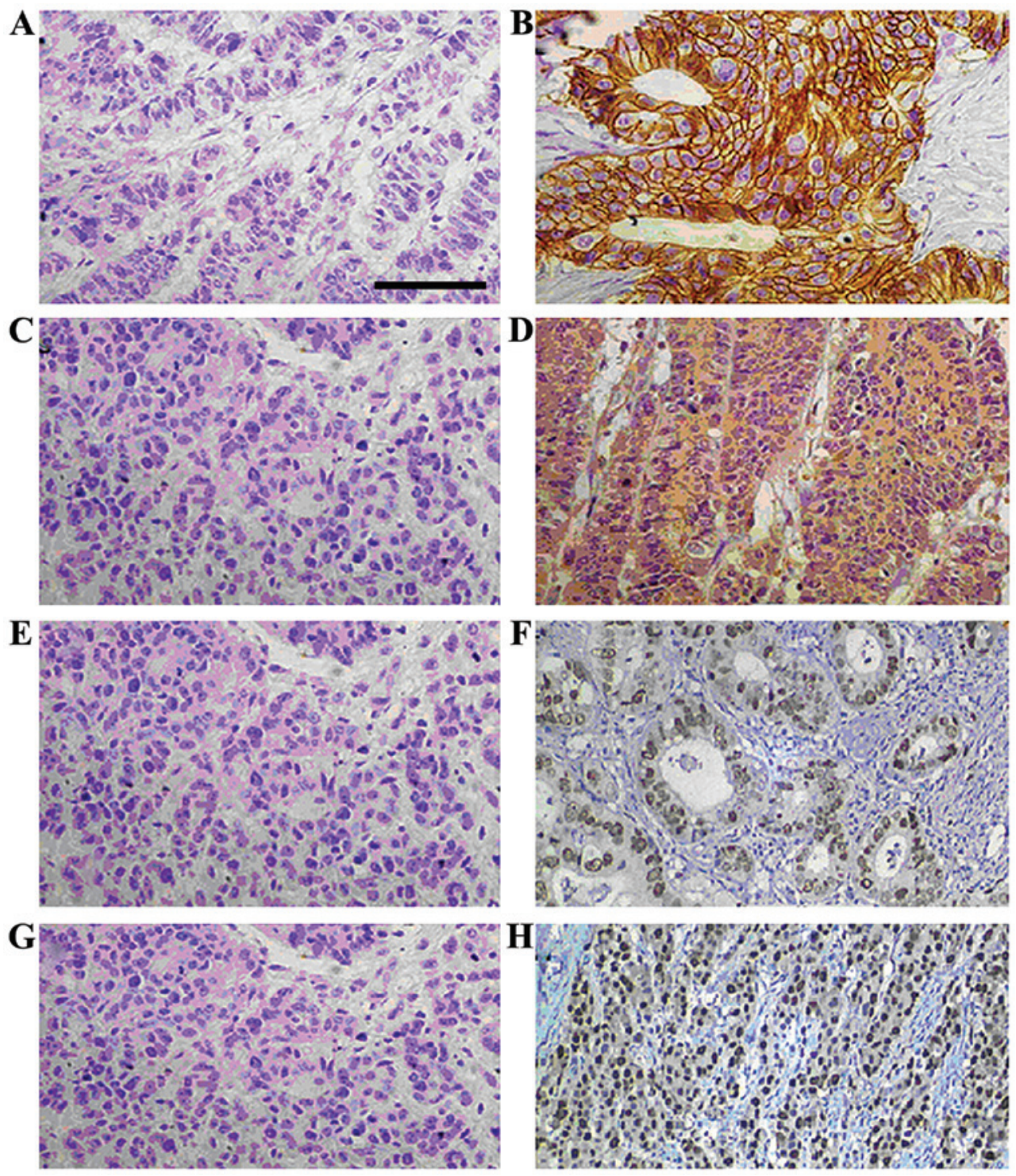

Figure 1. Immunohistochemical results of gastric cancer samples. (A) Tspan-1-negative, (B) Tspan-1-positive, (C) VEGF-negative, (D) VEGF-positive, (E) E-cadherin-negative, (F) E-cadherin-positive, (G) N-cadherin-negative and (H) N-cadherin-positive. Magnification, $\mathrm{x} 400$; scale bar, $50 \mu \mathrm{m}$. VEGF, vascular endothelial growth factor.

diameter, the mRNA expression levels of Tspan-1, VEGF and $\mathrm{N}$-cadherin were increased, while that of E-cadherin was decreased (Table II).
Correlation analysis. In immunohistochemical results, the positive rate of Tspan-1 was positively correlated with VEGF and $\mathrm{N}$-cadherin $(\mathrm{r}=0.426, \mathrm{P}=0.013 ; \mathrm{r}=0.521, \mathrm{P}=0.009)$, and 
Table II. Analysis of PCR results.

\begin{tabular}{lcccc}
\hline Item & Tspan-1 & VEGF & E-cadherin & N-cadherin \\
\hline Male & $0.4625 \pm 0.1325$ & $0.3659 \pm 0.1324$ & $0.1235 \pm 0.0685$ & $0.4857 \pm 0.1526$ \\
Female & $0.4526 \pm 0.1426$ & $0.3529 \pm 0.1268$ & $0.1325 \pm 0.0527$ & $0.4759 \pm 0.1637$ \\
Age, years & & & & \\
$<62$ & $0.4429 \pm 0.1258$ & $0.3652 \pm 0.1127$ & $0.1426 \pm 0.0737$ & $0.4659 \pm 0.1527$ \\
$\geq 62$ & $0.4725 \pm 0.1529$ & $0.3528 \pm 0.1235$ & $0.1258 \pm 0.0638$ & $0.4925 \pm 0.1649$ \\
Stage II & $0.3529 \pm 0.1123$ & $0.3251 \pm 0.1426$ & $0.1952 \pm 0.0859$ & $0.3325 \pm 0.1323$ \\
Stage III & $0.5214 \pm 0.1865$ & $0.3956 \pm 0.1568$ & $0.0965 \pm 0.0123$ & $0.5968 \pm 0.1527$ \\
Tumor diameter, cm & & & & $0.1857 \pm 0.0785$ \\
$<3.3$ & $0.4215 \pm 0.1238$ & $0.3123 \pm 0.1257$ & $0.0865 \pm 0.0232$ & $0.4215 \pm 0.1538$ \\
$\geq 3.3$ & $0.4968 \pm 0.1857$ & $0.4214 \pm 0.1869$ & $0.5263 \pm 0.2123$ \\
\hline
\end{tabular}

VEGF, vascular endothelial growth factor.

Table III. Analysis of survival prognosis.

\begin{tabular}{|c|c|c|c|c|c|c|c|c|}
\hline \multirow[b]{2}{*}{ Item } & \multicolumn{2}{|c|}{ Tspan-1 } & \multicolumn{2}{|c|}{ VEGF } & \multicolumn{2}{|c|}{ E-cadherin } & \multicolumn{2}{|c|}{ N-cadherin } \\
\hline & Positive & Negative & Positive & Negative & Positive & Negative & Positive & Negative \\
\hline \multicolumn{9}{|l|}{ Stage II $(n=84)$} \\
\hline $\begin{array}{l}\text { Median survival time, } \\
\text { months }\end{array}$ & 23.4 & 28.7 & 22.6 & 29.3 & 32.5 & 24.7 & 19.8 & 30.6 \\
\hline Survival rate, $\%$ & 42.5 & 65.9 & 37.1 & 63.3 & 70.0 & 40.7 & 35.1 & 57.4 \\
\hline \multicolumn{9}{|l|}{ Stage III $(n=66)$} \\
\hline $\begin{array}{l}\text { Median survival time, } \\
\text { months }\end{array}$ & 14.6 & 21.2 & 12.9 & 20.4 & 22.3 & 14.8 & 10.7 & 19.8 \\
\hline Survival rate, $\%$ & 25.5 & 52.6 & 30.0 & 65.4 & 58.3 & 24.1 & 27.9 & 56.5 \\
\hline
\end{tabular}

negatively related to E-cadherin $(\mathrm{r}=0.467, \mathrm{P}=0.011)$. In PCR results, the mRNA level of Tspan-1 was positively correlated with VEGF and N-cadherin $(\mathrm{r}=0.442, \mathrm{P}=0.011 ; \mathrm{r}=0.557$, $\mathrm{P}=0.006)$, and negatively related to E-cadherin $(\mathrm{r}=0.482$, $\mathrm{P}=0.008)$.

Analysis of survival prognosis. Among patients with stage II/III advanced gastric cancer, those with positive expression of Tspan-1, VEGF and N-cadherin had lower median survival time and survival rates than patients with negative expression, while patients with positive expression of E-cadherin had higher median survival time and survival rates than those with negative expression $(\mathrm{P}<0.05)$ (Table III).

\section{Discussion}

The present study showed that Tspan-1 plays important roles in cell signaling, adhesion regulation, metastasis, differentiation, proliferation and tumor cell immune escape (15). According to results of the study, the positive expression rates of Tspan-1, VEGF, E-cadherin and N-cadherin were 58.0, 50.0, 28.0 and $53.3 \%$, respectively in patients with advanced gastric cancer. The positive expression and mRNA levels of Tspan-1, VEGF, E-cadherin and N-cadherin was not correlated with sex or age, but associated with the cancer state (stage II or stage III) and maximum tumor diameter. With the increase of cancer stage and tumor diameter, the positive rates and mRNA levels of Tspan-1, VEGF and N-cadherin were increased, while those of E-cadherin were decreased. It has been demonstrated that VEGF is involved in the mechanism of tumor angiogenesis. Tumor proliferation, invasion, migration, relapse and other processes are inseparable from the nutrition support of blood microcirculation. VEGF is a strong cytokine regulating angiogenesis (16). E-cadherin and $\mathrm{N}$-cadherin are important molecular markers of EMT, of which E-cadherin is a marker of epithelial phenotype and N-cadherin is a marker of mesenchymal phenotype. The expression of the marker of epithelial phenotype decreased while that of the marker of mesenchymal phenotype increased. This is conssistent with the EMT occurrence and tumor metastasis. For malignant tumors of epithelial origin such as gastric cancer, the EMT occurrence plays major roles in tumor recurrence and metastasis (17). 
A further correlation analysis showed that the positive rate and mRNA level of Tspan-1 were positively correlated with VEGF and N-cadherin, and negatively related to E-cadherin. Among patients with stage II and III advanced gastric cancer, those with positive expression of Tspan-1, VEGF and $\mathrm{N}$-cadherin had lower median survival time and survival rates than patients with negative expressions, while patients with positive expression of E-cadherin had higher median survival time and survival rates than those with negative expression. This suggests that the high expression of Tspan-1 gene is associated with the TNM staging of patients with advanced gastric cancer and the tumor diameter influencing the survival prognosis, and may involve the processes of angiogenesis and EMT. Tspan-1 shows potential to become a target of early clinical diagnosis, intervention and prognosis evaluation. Therefore, additional sample size is needed, and the follow-up time should be extended to validate the conclusion.

\section{References}

1. Yung KW, Yung TT, Chung CY, Tong GT, Liu Y, Henderson J, Welbeck D and Oseni S: Principles of cancer staging. Asian Pac J Surg Oncol 1: 1-16, 2015.

2. Zheng YF, Tan LK, Tan BH, Sterling H and Kane R: Principles of surgical oncology. Asian Pac J Surg Oncol 1: 17-26, 2015.

3. Ghoneum M, Felo N, Nwaogu OM, Fayanju IY, Jeffe JA and Margenthaler DB: Clinical trials in surgical oncology. Asian Pac J Surg Oncol 1: 73-82, 2015.

4. Hi G, Dong M, Sheng W, Zhou J, Yu D and Sun W: Expression and clinical significance of Tspan 1 and Integrin $\alpha 6$ in human pancreatic ductal adenocarcinoma. Zhonghua Wai Ke Za Zhi 52: 781-786, 2014 (In Chinese).

5. Scholz CJ, Kurzeder C, Koretz K, Windisch J, Kreienberg R, Sauer G and Deissler H: Tspan-1 is a tetraspanin preferentially expressed by mucinous and endometrioid subtypes of human ovarian carcinomas. Cancer Lett 275: 198-203, 2009.

6. Chen L, Zhu YY, Zhang XJ, Wang GL, Li XY, He S, Zhang JB and Zhu JW: TSPAN1 protein expression: A significant prognostic indicator for patients with colorectal adenocarcinoma. World J Gastroenterol 15: 2270-2276, 2009.
7. Chen L, Yuan D, Wang GL, Wang Y, Wu YY and Zhu J: Clinicopathological significance of expression of Tspan-1, Jab1 and p27 in human hepatocellular carcinoma. J Korean Med Sci 25: 1438-1442, 2010.

8. Hölters S, Anacker J, Jansen L, Beer-Grondke K, Dürst M and Rubio I: Tetraspanin 1 promotes invasiveness of cervical cancer cells. Int J Oncol 43: 503-512, 2013.

9. Desouki MM, Liao S, Huang H, Conroy J, Nowak NJ, Shepherd L, Gaile DP and Geradts J: Identification of metastasis-associated breast cancer genes using a high-resolution whole genome profiling approach. J Cancer Res Clin Oncol 137: 795-809, 2011.

10. Cao J, Yang JC, Ramachandran V, Arumugam T, Deng DF, Li ZS, Xu LM and Logsdon CD: TM4SF1 regulates pancreatic cancer migration and invasion in vitro and in vivo. Cell Physiol Biochem 39: 740-750, 2016.

11. Wang P, Bao W, Zhang G, Cui H and Shi G: Transmembrane-4-L-six-family-1, a potential predictor for poor prognosis, overexpressed in human glioma. Neuroreport 26: 455-461, 2015.

12. Nabokina SM, Senthilkumar SR and Said HM: Tspan-1 interacts with the thiamine transporter-1 in human intestinal epithelial cells and modulates its stability. Am J Physiol Gastrointest Liver Physiol 301: G808-813, 2011.

13. Jiang $\mathrm{X}$, Zhang $\mathrm{J}$ and Huang Y: Tetraspanins in cell migration. Cell Adhes Migr 9: 406-415, 2015.

14. Lu Z, Luo T, Nie M, Pang T, Zhang X, Shen X, Ma L, Bi J, Wei G, Fang $G$ and Xue X: TSPAN1 functions as an oncogene in gastric cancer and is downregulated by miR-573. FEBS Lett 589: 1988-1994, 2015.

15. Scholz CJ, Sauer G and Deissler H: Glycosylation of tetraspanin Tspan-1 at four distinct sites promotes its transition through the endoplasmic reticulum. Protein Pept Lett 16: 1244-1248, 2009.

16. Sciuto TE, Merley A, Lin CI, Richardson D, Liu Y, Li D, Dvorak AM, Dvorak HF and Jaminet SC: Intracellular distribution of TM4SF1 and internalization of TM4SF1-antibody complex in vascular endothelial cells. Biochem Biophys Res Commun 465: 338-343, 2015.

17. Dai J, Qian C, Su M, Chen M and Chen J: Gastrokine-2 suppresses epithelial mesenchymal transition through PI3K/ AKT/GSK3 $\beta$ signaling in gastric cancer. Tumour Biol 37: 12403-124010, 2016. 